1 | 2004

NOVECENTO... E DINTORNI

Dire la guerre?

\title{
Gadda: la scrittura come «strazio del passato continuo»
}

Manuela Bertone

\section{(2) OpenEdition}

Journals

Edizione digitale

URL: http://journals.openedition.org/cei/233

DOI: $10.4000 /$ cei.233

ISSN: 2260-779X

Editore

UGA Éditions/Université Grenoble Alpes

\section{Edizione cartacea}

Data di pubblicazione: 15 novembre 2004

Paginazione: 55-71

ISBN: 978-2-84310-057-4

ISSN: 1770-9571

Notizia bibliografica digitale

Manuela Bertone, «Gadda: la scrittura come «strazio del passato continuo»», Cahiers d'études italiennes [Online], 1 | 2004, online dal 15 mai 2006, consultato il 27 mars 2021. URL: http://

journals.openedition.org/cei/233 ; DOI: https://doi.org/10.4000/cei.233 
GADDA

LA SCRITTURA COME «STRAZIO DEL PASSATO CONTINUO »

\author{
Manuela Bertone \\ Université de Savoie (Chambéry)
}

Dirò della guerra di Gadda e di quella che chiamo, con formula parzialmente continiana, la scrittura come "strazio del passato continuo ${ }^{1}$ ", partendo da alcune osservazioni che Carla Benedetti fa in Il tradimento dei critici, libro coraggioso e controverso che denuncia le consuetudini mentali che hanno paralizzato la critica e descrive le false rappresentazioni che hanno dominato la vita culturale italiana degli ultimi decenni. A Gadda, la Benedetti dedica ampio spazio per due ragioni: anzitutto perché egli possedeva "il senso del complesso", ovvero una visione forte che rifiutava i recinti, le chiusure, le sfere, le specializzazioni; poi perché

\begin{abstract}
gli aspetti forti, brunianamente "eroici» del suo pensiero sono stati quasi del tutto tralasciati dai suoi canonizzatori. E anche questa ricezione "astraente» della sua opera è un ulteriore esempio del tradimento dei critici. Credo che la cultura italiana abbia un conto in sospeso con questo scrittore, il più lodato e il più frainteso d'Italia. E non per mancata valorizzazione della sua opera, che è stata anzi affidata alla cura scrupolosa dei migliori filologi e celebrata unanimemente come pezzo sommo della letteratura del Novecento; ma per la crudele parcellizzazione a cui è stata sottoposta in decenni di letture parziali, locali e frantumanti. Come per un difetto di messa a fuoco o, forse, per un sordo rifiuto, l'opera di Gadda è stata ripetutamente privata della sua drammatica interezza. Si è lavorato di forbici, e Gadda è ora una figurina incollata in un album. Dietro la sua immagine ritagliata non c'è nessun fondale: non montagna o trincea; non centrale idroelettrica o camera d'affitto; non cortei di reduci per le piazze d'Italia $[\ldots]$

Di Gadda si ama il pezzo, la bella pagina, la virtuosistica lingua, la scrittura espressionistica, il plurilinguismo, se ne studiano le fonti letterarie e pittoriche, la formazione trasversale scientifica e umanistica, si studia la travagliata vicenda di scrittura e riscrittura dei suoi testi... Ma si perde di vista il collante che unisce tutti quei frantumi. Si perde di vista il loro «nesso di organicità »- come lo chiamerebbe Gadda. [...] il tutto sfugge così come sfuggiva al «ceto mercativo-politecnico" della "tribù» milanese in cui gli era capitato di nascere. E come sfuggiva ai
\end{abstract}

1. G. Contini, Introduzione alla "Cognizione del dolore», ora in Quarant'anni d'amicizia, Torino, Einaudi, 1989, p. 22 : «E' semmai nel poème en prose [...] che lo strazio del passato continuo può giungere a interrompersi in memoria discreta, e dunque in immagine, e perciò stesso in dolcezza d'immagini». 
generali, ai governanti e all'individuo italico, da cui Gadda sentiva oltraggiata quella saggezza e quella pulsione di vita che viene solo dal poter pensare la complessità ${ }^{2}$.

Sulla guerra in Gadda e di Gadda (la guerra '15-'18) si è scritto e detto molto, ma non si è insistito abbastanza sul fatto che la guerra non è soltanto un elemento del discorso visibile nella tessitura dei contenuti. Gadda ha raccontato la guerra: nel Giornale di guerra e di prigionia nel Castello di Udine, in parte nel Racconto italiano, nella Meccanica, in molti racconti. La guerra è pertanto un tema che consente di esplorare uno dei luoghi mentali in cui si ritrova l'opera di Gadda nella sua «drammatica interezza » : la guerra è il "collante», è il fondale fatto di «montagna o trincea» su cui la Benedetti ha posto l'accento e su cui, credo, Gadda conta saldamente per "pensare la complessità ».

Ma la guerra non è solo un tema, è un modo di scrivere, è una situazione di scrittura, una curvatura del discorso, è un modo di essere-nellascrittura che non riguarda solo le pagine specificamente dedicate a quell'evento, ma affiora nell'intera distesa testuale. La centralità e la ricorrenza costituiscono criteri sufficienti per giustificare un'interpretazione. Pertanto proprio questo vorrei proporre: un'interpretazione, ovvero una ricerca nel testo (e non altrove), un'operazione di unificazione di ciò che, se non interpretato, permarrebbe solo disperso nei testi.

Mi concedo una cauta incursione introduttiva nell'extratestuale, visto che i testi di cui parlerò l'autorizzano e addirittura la richiedono, poiché vi è un'extratestualià che sta dentro al testo e come tale va compresa. E' infatti utile ricordare che la prima guerra costituisce per Gadda un investimento smisurato - come del resto per molti uomini della sua generazione, convinti com'erano di partecipare al coronamento di un'impresa eroica che idealmente avrebbe portato a compimento il Risorgimento -. Per Gadda la guerra sarà non un'occasione qualunque, bensì l'occasione per procedere alla propria ricostruzione identitaria, alla ricostituzione di sé. E' come se la divisa gli servisse a tener cuciti i brandelli compositi di un'identità che si era andata formando per frammenti contraddittorî, per niente simili alle tessere di un mosaico o di puzzle destinate a disporsi in un tutto coerente. Quel che Gadda chiedeva alla divisa da alpino era trasformare in vita vera la sua vita modesta, spenta, deludente. Al contrario di Renato Serra, che dalla guerra non si aspettava nulla, dalla guerra Gadda si aspettava ed esigeva tutto, e per sé e per la Patria. Per Gadda la

2. C. Benedetti, Il tradimento dei critici, Torino, Bollati Boringhieri, 2002, p. 23-25. 
Patria è "la religione degli avi [...] la religione del popolo italiano ${ }^{3}$ » e quindi la guerra è sì "necessaria», ma anche "santa ${ }^{4}$ ».

L'ideale del soldato Gadda è quello di « una vivente Patria, come nei libri di Livio e di Cesare " : la Patria è, ma al contempo la Patria è in divenire; la Patria è insieme realizzazione compiuta e progetto. La guerra è precisamente l'evento che concilia, riunifica e rappresenta queste esigenze: l'essere e l'idea, la compiutezza e il divenire, il fatto e il farsi. Gadda lo dirà nella Meditazione, quando sceglierà il punto di vista del soldato, del combattente (di gran lunga il più accreditato in questo suo lavoro filosofico), per spiegare il funzionamento del sistema del reale e più precisamente il nucleo etico che caratterizza un sistema funzionante. Un sistema funzionante è un sistema autoregolato emendatosi e potenziatosi di gruppi logici coinvolgibili. Il bene consiste nel raggrupparsi e diramarsi di relazioni infinite, il male invece nel raggrumarsi di un unico significato, che dissipa le relazioni infinite e mortifica il flusso creativo. Gadda esprime questo viluppo con una formula tutta sua: intervenire nel sistema « $\mathrm{n} »$ (acquisito) per approdare, arricchendolo, al sistema « $\mathrm{n}+1 »$ (acquisendo), significa impedire l'avvento del male, inteso come regresso dall'adempimento. Arrestarsi ad « $\mathrm{n}$ » significa accettare un equilibrio trofico, tralasciare la possibilità di ascendere verso « $\mathrm{n}+1$ ", verso l'ignoto, verso gli infiniti aggrovigliamenti che costituiscono il sistema. Ora, ci dice Gadda, il soldato va in guerra sacrificando « $\mathrm{n}$ " (il proprio sentimento eroico) per tendere ad «n $+1 »$ (il divenire della patria), cioè per tendere a un fine che è un meglio.

Non sarà un caso se Gadda istituisce addirittura un vincolo analogico fra la sua guerra e quelle di Cesare. In un recente intervento su Gadda e il culto di Roma ${ }^{6}$, ho evocato un lacerto del Racconto italiano, in cui Gadda, schizzando il profilo del tenente Tolla, scrive: «La lettura di Cesare lo aveva profondamente appassionato tanto che aveva pensato di scrivere lui pure dei commentari, ma gli mancava la guerra delle Gallie ${ }^{7}$ ». Roscioni, nella sua biografia, osserva che è proprio la guerra delle Gallie che smette

3. Con queste parole lo zio Giuseppe Gadda, senatore e ministro, presenta in Ricordi e impressioni $\mathrm{i}$ momenti forti della lotta risorgimentale (cit. in G. Roscioni, Il Duca di Sant'Aquila, Milano, Mondadori, 1997, p. 14).

4. C.E. Gadda, Giornale di guerra e di prigionia, in Saggi giornali favole II, Milano, Garzanti, 1992, p. 533.

5. C.E. Gadda, Dal castello di Udine verso i monti, in Il castello di Udine, in Romanzi e racconti $\mathrm{I}$, Milano, Garzanti, 1988, p. 152.

6. M. Bertone, "Mirabilia Urbis Romae": le culte de Rome dans l'oeuvre de Gadda, in Rome. Myhtes et symboles, études réunies par B. Toppan, PRISMI, Université Nancy 2, 2001 (ma 2003), p. 243-266.

7. C.E. Gadda, Racconto italiano di ignoto del Novecento, in Scritti vari e postumi, Milano, Garzanti, 1993 , p. 450. 
di mancare al tenente Gadda nel '15; infatti, egli scriverà il proprio Giornale seguendo lo stile degli antichi commentari. Ma anche le pagine dell'ex-tenente Gadda, ormai bizzoso capitano in congedo (penso per esempio a Tecnica e poesia e a Il dolce riaversi della luce) rinviano volentieri al proconsole, ai "commentari di Cesare, tanto per dare a Cesare quel che gli spetta ${ }^{8}$ ». Parlando della sua guerra, Gadda si rifa continuamente al genio militare di Cesare, alle azioni militari della $X$ Legio, al testo del $D e$ bello Gallico, come se si trattasse di fonti di ispirazione ancora vive e utili per il suo tempo, alle quali gli ufficiali italiani avrebbero quindi fatto bene a ispirarsi per elaborare tattiche efficaci. Non conoscendo la strategia di Cesare, il suo libro, i suoi dettami, i responsabili militari italiani, da «naufraghi del latino ${ }^{9}$ » quali erano, si sono dimostrati incompetenti nel '15'18.

Ricordato anche questo, ricordato cioè che l'ispirazione patriottica e guerriera di Gadda ha radici tutt'altro che effimere nella storia e che si fonda sulla costante attualizzazione di gloriose imprese militari antiche, vorrei riportare uno stralcio del giudizio dato su Gadda dalla commissione di inchiesta che lo interrogò, come altri ufficiali reduci dai campi tedeschi, al ritorno dalla prigionia. Ecco il "profilo dell'inquisito":

\footnotetext{
In apparenza saldo e robusto [...]. Ha buona cultura e si interessa molto ai doveri militari. Dimostra elevati sentimenti. Fu decorato di medaglia di bronzo al valore per fatto d'arme sul Passo Faiti (18-23 agosto 1917). E' da ritenersi ufficiale di ottimo rendimento, se potrà rimettere i suoi nervi in perfetta calma, ora alquanto scossi per le speciali condizioni della sua famiglia 10 .
}

Noi sappiamo di trovarci di fronte a un ritratto dell'artista da giovane (che poi è anche una scheda segnaletica del soggetto narrante gaddiano) che fissa i tratti di un profilo psicologico che negli anni rimarrà immutato : sappiamo che i nervi di Gadda non ritroveranno mai la perfetta calma, che per sempre rimarrà scosso come allora, senza mai rassegnarsi alla perdita del fratello Enrico e facendo delle "speciali condizioni di una famiglia» il motivo portante di tutti i suoi scritti. Spiritualmente, Gadda non si allontanerà mai dai luoghi e dagli eventi della guerra. La sua dipendenza dai momenti di allora è profonda e intensa. Nel 1938, scrivendo all'amico Tecchi (futuro dedicatario del Giornale), ricorda la guerra come « il tempo dell'entusiasmo e del dolore: e di quel terribile sacrificio che le nuove

8. C.E. Gadda, Tecnica e poesia, in Gli anni, in Saggi giornali favole I, Milano, Garzanti, 1991, p. 244.

9. C.E. Gadda, Elogio di alcuni valentuomini, in Il castello di Udine, cit., p. 132.

10. Cit. da D. Isella nella "Nota al testo» riferita al Giornale di guerra e di prigionia, in Saggi giornali favole II, cit., p. 1119. 
generazioni non conoscono». Nel 1939 gli dice: «Noi abbiamo vissuto una spaventosa guerra, martirio e orgoglio che altri non ha conosciuto". Ripetutamente traccia una linea di demarcazione emotiva che lo separa dagli altri e dalla storia contemporanea: vede soltanto la sua storia e il suo dolore, il suo martirio e il suo orgoglio, dimentico perfino del fatto (siamo nel '38-'39) che per le nuove generazioni si sta preparando un sacrificio enorme, che si preannuncia già di dimensioni almeno analoghe a quello vissuto dalla sua. Ma il suo tempo interiore non passa e gli anni che lo separano dalla sua guerra sono tutti raggrumati attorno a quella prova: rivelatore in proposito il lapsus in cui incorre nel datare « 9 ottobre 1915" una lettera a Tecchi del 9 ottobre $1931^{11}$.

Che dall'epoca della sua guerra Gadda abbia scritto stando fuori da quello che chiamiamo il tempo reale, filtrando tutta la percezione delle cose e la conoscenza del mondo attraverso quell'evento e la propria ossessiva ricostruzione di quell'evento, lo dimostra un suo testo (praticamente sconosciuto fuori dalla cerchia dei gaddisti), pubblicato nel novembre 1939 su "Le vie d'Italia", Le Marine da guerra delle Nazioni belligeranti... e le loro forze militari terrestri. Si tratta di uno degli otto pezzi scritti fra il '36 e il ' 45 non compresi nemmeno nella monumentale edizione Garzanti per motivi che non andremo a sondare adesso, ma la cui esclusione serve almeno a spiegare perchè si tratta di un pezzo che praticamente quasi nessuno ha letto. Come dice il titolo, dovrebbe trattarsi di un intervento dedicato a un argomento di attualità. Siamo nel 1939, l'Italia non è ancora entrata in guerra. Ritroviamo il Gadda cultore del dettaglio minuto, capace di recensire a lungo, e tonnellata per tonnellata, la potenza navale dei paesi belligeranti, nonché il Gadda che si distoglie senza preavviso dal tema portante del discorso per parlare d'altro. Improvvisamente, un sottotitoletto informa che l'autore, che fino ad allora si era occupato degli armamenti del presente, si dedicherà ora all'argomento «Dalle opere belliche dei Romani alle linee Sigfrido e Maginot». Dopo di che, due righe scarse sono dedicate alle linee Sigfrido e Maginot, semplice pretesto per lanciare « una rievocazione degli antichi sistemi di fortificazione in uso presso i Romani ». In altre parole, Gadda si occupa del solo lavoro militare che considera "grandioso"; si libera del tono leggermente burocratico e della cadenza elencativa che caratterizzano la prima parte dell'articolo e passa in rivista con passione e dettagli «la saldezza e la vastità delle concezioni romane». L'ingegnere tutto preso dalla guerra delle Gallie, ovvero

11. Le citazioni tratte dal carteggio Gadda-Tecchi sono reperibili in C.E. Gadda, $A$ un amico fraterno. Lettere a Bonaventura Tecchi, Milano, Garzanti, 1984. 
dal progetto della sua guerra quale avrebbe dovuto essere (modellato appunto sull'esempio di quella delle Gallie), passa in rassegna le mura, le fortificazioni campali, il limes dell'impero, la guerra di Giudea, approdando all'exploit di Cesare che sconfigge i Galli. Questo excursus, che sembra esserci recapitato da un altro mondo, da un altro tempo, costituisce di fatto un esempio di brusca e obliqua transizione dal narrato al vissuto, di innesto nel narrato di eventi in apparenza lontani, e dunque documenta una frattura il cui effetto è straniante, ma che in realtà attesta il travaso continuo di un certo passato nel presente, di un passato che fa da sostegno forte per un presente debole. Se Gadda è vuoi dimentico vuoi dissacratore della storia è perché per lui la storia vera è quella che si è vista sui campi di battaglia.

Raffaele Donnarumma ha sottolineato che Gadda si mette «in parentesi di fronte alla storia" per parlarne più che come portatore di un'esperienza, come uno che sia di fronte o al di sopra degli eventi, ma non negli eventi : "la sola forma di partecipazione alla storia fu per lui l'esserne travolto; e la sola forma di etica il non lasciarsene travolgere ${ }^{12}$ ». Pier Paolo Pasolini ha rilevato in questo tratto " una fissazione narcissica implicante, oltre la naturale deformazione del rapporto gnoseologico con la realtà, una nostalgia (non crepuscolare) per l'epoca in cui tale fissazione s'è avuta ${ }^{13}$ ": Gadda scrive sempre da soldato e di soldati, di chi è stato soldato, di chi è in età da esserlo; di madri, di spose, di vedove, di nuore, di fidanzate, di figlie di soldati. Ha ragione Donnarumma quando afferma che tutto questo accade non tanto perché Gadda si fissa su un momento della sua esistenza di cui fa un uso autobiografico, bensì perché di quel momento fa un uso intellettuale ed etico. Quel momento è il garante dell'unità testuale, dà valore alla scrittura e la scrittura, in Gadda, non vuole dar spazio a destini individuali che non siano destini collettivi: «non iscrivo per me, scrivo perché salti fuori qualche cosa che possa valere a farci più forti e più avveduti in ogni futura contingenza, nelle distrette del male ${ }^{14}{ }$. In questi termini Gadda dà la spiegazione che fissa nella nostra mente il senso di quel nesso tra evento e testo:

la descrizione, il desiderio di conoscere e di approfondire, si estese per gradi, specie con la guerra (1915-1918), all'indole e ai tipi e al destino degli umani, ai rapporti fra le creature: la vita militare e il servizio in guerra sono una trama continua di rapporti, sull'ordito combinatorio del destino: il sibilo che stende a terra, vicino a me, il mio compagno non può lasciarmi indifferente alla contemplazione della morte, alla mortale probabilità di essere suo commili-

12. R. Donnarumma, Gadda. Romanzo e pastiche, Palermo, Palumbo, 2001, p. 17.

13. P.P. Pasolini, Gadda, in Passione e ideologia, Torino, Einaudi, 1985, p. 277.

14. C.E. Gadda, Impossibilità di un diario di guerra, in Il castello di Udine, cit., p. 141. 
tone anche nel regno delle ombre. Così la mia scrittura, dapprima nei diari e nelle lettere [...], veniva a investire la vicenda umana, la storia delle anime ${ }^{15}$.

La guerralcosì la mia scrittura - siamo nel 1951: ecco lo strazio del passato continuo fissato nell'eterno presente del «sibilo che stende a terra, vicino a me, il mio compagno", qui, ora.

Analizzando Il castello di Udine, ho messo in evidenza un certo numero di conflagrazioni temporali simili a quelle evocate sopra, usando ad analisi ultimata la definizione di "allucinazione descrittiva» e precisando che le parole "castello di Udine", che fanno da titolo ad un intero volume dedicato in piccola parte al castello di Udine, si riferiscono solo di striscio al luogo che designano, mentre denotano pienamente lo stato d'animo che incombe su tutti gli scritti gaddiani (Meditazione milanese compresa), caratterizzati come sono dalla necessità di riattivare la comunicazione con il passato dello scrittore-combattente ${ }^{16}$. Raggruppando sotto l'insegna di quel certo nome un fascio di scritti disparati, nati in epoche diverse e di contenuto vario, Gadda dà una speciale curvatura al proprio modo di fare scrittura: quella curvatura che avevo chiamato «scrittura del castello». Il "castello" cessa di essere contemplato come luogo-emblema di se stesso o di un fatto storico preciso, per essere coinvolto in un vasto circuito di inquietudini e fissazioni, e serve ad additare il materializzarsi della scrittura come nucleo espressivo costituito dalla conflagrazione fra un passato intruso a forza nel presente ovvero come dichiarazione dell'impossibilità di risolvere armonicamente il loro rapporto.

Di «cortocircuito temporale» parla Lucio Lugnani nell'esaminare gli affioramenti testuali dell'evento-guerra in scene narrative che nulla hanno a che vedere, in apparenza, con quell'evento. Ecco uno degli esempi più significativi proposti da Lugnani, tratto dall'Adalgisa:

Nel morir tisica, poi, era inarrivabile. Fosse che qualche volta eravamo magari mezzo storditi, bevuti non direi, poveracci, non ricordo bene,... ora,... certo è che il mal sottile tra la nuvolaglia de' veli, sottintendeva in lei un seno, un davanti... già... di povera creatura consumata dalla clorosi... oh! quest'è certo... ma una clorosi da secondo impero, date retta..., che ne avanzava pur sempre qualche cosa di potabile... ve lo garantisco io...

Il tragico estinguersi di quella vita era a momenti più vero e immediato del nostro ardore. «Oh Dio! compermèss che me ven fastidi", sospirava Remigio come svenendo : e tutti gli facevano largo dassenno, qualcuno zittiva, furibondo : e, allora, un bieco muggire di proteste a catena, di minacce. Che poi ci lasciò la pelle per davvero, sul Podgora ${ }^{17}$.

15. C.E. Gadda, Intervista al microfono, in I viaggi la morte, in Saggi giornali favole I, cit., p. 502.

16. Vedi M. Bertone, Il romanzo come sistema. Molteplicità e differenza in C.E. Gadda, Roma, Editori Riuniti, 1993.

17. C.E. Gadda, L'Adalgisa, in Romanzi e racconti I, cit., p. 531-532. 


\section{Manuela Bertone}

Senza evocare per intero il commento critico, leggiamo le conclusioni di Lugnani :

Il narratore è impegnato a narrare la storia di Adalgisa, non quella di Remigio e non la propria; ma dal loquace e teatrale finto svenimento dell'amico, da quel fargli largo dassenno degli spettatori turbati e poi dal furore, dai muggiti biechi di protesta, dalle minacce degli altri, disturbati, la sua memoria è tratta a trascorrere, immediatamente, e irresistibilmente, con un nesso semplificato al massimo e più proprio al parlato che non alla sua discorsività letteraria ("Che poi»), a ben altre minacce, a più biechi muggiti, ad un altro furore, al decesso muto di Remigio e al suo lasciarci la pelle per davvero, sul teatro del Podgora. Ossia all'exitus di un'altra storia, che non è narranda e non sarà narrata. Non un fattore narratologico (la prolessi) e non la temporalità del racconto contano, ma la dolorosa, rabbiosa esperienza umana del tempo, che il soggetto narrante esprime quasi suo malgrado. E' da quest'ultima che riaffiora, come quello d'un affogato, il cadavere di Remigio, il quale, da morto, non appartiene propriamente a quest'ultimo disegno milanese dedicato ad Adalgisa, ma piuttosto al più vasto e folto cartone della memoria del personaggio-narratore ${ }^{18}$.

Un altro esempio istruttivo, altresì raccolto da Lugnani, si trova nelle pagine del Pasticciaccio, dove il commissario Ingravallo, dinanzi al cadavere straziato di Liliana, viene visto vedere la scena nel modo seguente:

Er sangue aveva impiastrato tutto er collo, er davanti de la camicetta, una manica: la mano: una spaventevole colatura d'un rosso nero, da Faiti o da Cengio (don Ciccio rammemorò subito, con un lontano pianto nell'anima, povera mamma!) ${ }^{19}$.

Ecco che don Ciccio ricorda le colate di sangue sul Faiti o sul Cengio, mentre il narratore esclama, in sua vece, «Povera mamma!». Si cancella il fatto di sangue, questo fatto di sangue, mentre nella storia irrompe la memoria del soldato Ingravallo e, con essa, altro sangue e un lutto che confluisce in quello presente, che riguarda una donna ("Povera mamma!») che non è la stessa che giace al suolo sotto gli occhi del commissario Ingravallo, ma che come "povera Liliana" è appena entrata in scena e va a dare, con questo suo essere "povera" come la successiva, una brusca sottolineatura a quella rottura del nesso temporale che seguirà e che poi altro non è che la particolare legatura di un nesso mentale che solo al soldato-commissario Ingravallo può appartenere (detto per inciso, Ingravallo è voluto coetaneo da Gadda, poichè nel 1927 - tempo della trama - ha come lui 34 anni, ed è per giunta memore del fatto d'armi in cui si svolse l'azione eroica che valse a Gadda la medaglia di bronzo al valor militare).

Che tratti di eventi o di persone, di oggetti, di elementi del paesaggio, del clima, Gadda sceglie la rappresentazione per conflagrazione, per cortocircuito. C'è chi, in questo fenomeno, ha visto un'inclinazione all'artificio

18. L. Lugnani, Del tempo. Racconto discorso esperienza, Pisa, ETS, 2003, p. 93.

19. C.E. Gadda, Quer pasticciaccio brutto de via Merulana, in Romanzi e racconti II, Milano, Garzanti, 1989, p. 58-59. 
e all'ornamento, l'elaborazione di una poetica ispirata, consapevolmente, ai modelli e alle prescrizioni di un'estetica barocca. Sostenere che Gadda riprende e fa sua la problematica del barocco storico, parlare di Gadda barocco (senza nemmeno usare le virgolette) significa prendere lucciole per lanterne. Siamo invece nel giusto se, come fa Robert Dombroski, le virgolette a "barocco ${ }^{20}$ " le mettiamo precisando che l'immaginazione barocca di Gadda ha senso soltanto se collegata con orientamenti intellettuali particolari, diversi da quelli del barocco storico. Aggiungiamo, parafrasando Gadda stesso, che barocco è il mondo che vede, ritrae e descrive durante la guerra e dopo la guerra: "barocco » è il mondo nel suo essere destabilizzato e destabilizzante, fatto di insensatezza e mancanze, schemi codificati inerti, ideali e progetti sottoposti a torsioni mostruose. Ascoltiamo lui: "in ambienza bugiarda, in circostanze corrotte, lo spirito dello scrittore è preso da un'angoscia, da un'unica: col suo segno, duro segno, reagire alla scioccaggine ${ }^{21} »$.

Già parlando di scrittura-del-castello avevo preferito evitare la categoria del barocco, temendo che Gadda mi potesse rinfacciare dall'aldilà che il barocco è per lo più "prezioseggiante stramberia ${ }^{22}$ ». Con Dombroski, ci siamo tante volte detti che quella categoria era diventata invasiva e, di fatto, pensavamo, bisognerebbe fare un passo indietro per farne, poi, uno avanti. Un passo indietro si può fare scegliendo un'etichetta dimessa, quasi di grado zero, e parlare molto semplicemente di «scrittura-guerra»: continuando a elaborare la metafora della conflagrazione, direi che sulla pagina di Gadda, al di qua come al di là del Castello di Udine, nulla è rappresentato pacificamente, vediamo tracce di conflitto, di ferite e lesioni, lo scontro, il dissidio, la battaglia che nascono dall'esplosione di una scintilla. Vediamo insomma l'evento che si dispiega mentre osserviamo «il trauma che legge il mondo esterno filtrandolo attraverso le sue ossessioni $^{23}$ "; vediamo dispiegata, continuamente riattivata e rimessa in circolo quella che Gadda stesso chiama, dalle pagine del Castello, «la frenesia bellica del nostro".

Che cosa accade, di preciso?

Torniamo ai termini della Meditazione. La guerra rivela a Gadda che « $\mathrm{n}+1 »$, il bene, è un'aspirazione impossibile; l'ideale, un progetto irrealizzabile: in guerra, scopre che « $\mathrm{n}$ ", la stasi, ed «n-1», il regresso, sono le

20. R.S. Dombroski, Gadda e il barocco, Torino, Bollati Boringhieri, 2002, p. 7.

21. C.E. Gadda, Come lavoro, in I viaggi la morte, cit., p. 435.

22. C.E. Gadda, Fatto personale... o quasi, in I viaggi la morte, cit., p. 499.

23. R. Donnarumma, cit., p. 128. 
uniche esperienze fruibili e altresì le uniche esperienze comunicabili. « $\mathrm{n}$ » ed «n-1» sono il male, danno dolore, sono dolore, sono il dolore. Da allora, la scrittura non fa che inscenare il trauma di quella scoperta; e lo fa esibendo l'illusione, rinvangando il progetto virtuoso di ascesa verso l'ideale, sondando le aspirazioni, testimoniandone il crollo, ricercando le cause del male. Scrivere, ci dirà Gadda, è conoscere, procedere dal noto verso l'ignoto (quindi è in sé progetto etico), ma conoscere è andare a scoprire le cause del dolore, è aggiungere dolore. Poiché avvicinarsi al reale per conoscerlo significa scoprire che non c'è nessuna pienezza nelle cose, non ci sono esiti catartici, ma soltanto disordine, inganni, eccessi, artifici, frantumi, guasti, lacerazioni, pasticcio. Gadda definisce, a fine percorso, il massimo grado di forza cognitiva cui può approdare il soggetto, nel voler capire e spiegarsi il "pasticciaccio ", e lo definisce " cognizione del dolore». Con la scrittura-cognizione del dolore ci si connette al mondo, si tenta per lo meno di dare una forma al dolore, ma oltre non è dato di andare: " cognizione del dolore è conoscenza del mondo nell'umano, pasticciaccio è lo stato del mondo in cui l'uomo è immerso e perduto come uomo ${ }^{24}$ ".

Quali fattezze assume, in concreto, la scrittura-guerra? Dovendo associare dei lineamenti alla scrittura di Gadda, Dombroski suggerisce quelli del Grido di Edvard Munch, precisando con Fredric Jameson, in termini leibniziani che Gadda avrebbe condiviso, che il quadro di Munch rappresenta "l'esperienza quintessenziale del "dolore indicibile all'interno della monade" ${ }^{25}$ ". Li avrebbe condivisi anche perché di sé dà una descrizione cui, a distanza di decenni e con un oceano di mezzo, le parole di Jameson sembrano fare eco: «La mia monade e il mio io sono delle baracche sconquassate rispetto alle pure sfere d'acciaio di Leibniz e hanno mille finestre e fessure ${ }^{26}$ ". Baracche sconquassate piene di finestre e fessure, curiosa immagine da opporre a quella delle sfere d'acciaio; e poi che c'entra con il dolore all'interno della monade? C'entra, perchè la baracca del campo militare, poi la baracca del campo di concentramento, decine di volte evocata, descritta, schizzata, perché decine di volte costruita, riparata, organizzata, è per Gadda il primo luogo di scrittura e, come sottolinea nel Giornale, mentre scrive in baracca, è l'unico vero luogo di raccoglimento

24. Id., p. 51

25. R.S. Dombroski, Gadda e il barocco, cit., p. 28. Dombroski si riferisce a F. Jameson, Il postomoderno, o la logica culturale del tardo capitalismo, Milano, Garzanti, 1989 [ed. orig. 1984].

26. C.E. Gadda, Meditazione milanese, in Scritti vari e postumi, cit., p. 227n. 
per il soldato e per il prigioniero: la baracca, rifugio precario, instabile, effimero, è il luogo dei «dolorosi pensieri».

La scrittura-guerra è pertanto la scrittura della baracca e del baraccato, di colui che fu gran progettista e disegnatore di baracche, esperto misuratore di finestre e vetri, addirittura membro fondatore di una «società dei baraccani", sorta di cenacolo letterario improvvisato nella baracca del Lager, ma sempre minacciato e perseguitato dalle sgocciolature, dalle fessure, dalle aperture ai quattro venti, dallo sconquasso. Come ha recentemente sottolineato Federica Pedriali, Gadda scrive «da recluso-reietto subumano cui non è di alcuna consolazione l'ipotizzare, come invece fa per disperazione di prigioniero, che "se una libellula vola a Tokio innesca una catena di reazioni che raggiungono me" 27 ".

La scrittura-guerra assume, naturalmente, le fattezze del romanzesco, inteso come pura convenzionalità letteraria, come schema scontato, esausto, triviale, pretestuoso: nella Meditazione breve circa il dire e il fare Gadda dà "bugia», "consecuzione parolaia», "favolone ${ }^{28}$ ", come sinonimi di romanzo. L'autenticità etica della scrittura-guerra, della scrittura del baraccato, risiede altrove: nell'espressione, nello stile, nella tecnica, nella funzione autorale come garante dell'unità testuale che appunto sfilaccia le trame tessute dal codice letterario. Come precisa Dombroski, la monade scrive, scrive del mondo chiuso, sigillato nella propria interiorità. Il codice è praticamente una provocazione che Gadda accetta per denunciarla e ribellarvisi : per questo disse bene Contini quando, all'altezza del Castello di Udine, affermò che la scrittura di Gadda è una scrittura provocata, anziché provocatoria, reattiva quindi e non aggressiva, come alcuni vollero credere. "Ciascuno - dice Gadda - manovra nel suo campo ${ }^{29}$ ".

La scrittura-guerra è caratterizzata da un'impostazione conflittuale della pagina, divisa talora addirittura in due settori da uno sbarramento che separa due parti di testo poste a confronto: fra sé e chi legge e fra le due parti del testo, testo annotato e testo annotativo, per esempio, fra autore del testo e autore delle chiose viene scavata una trincea che delimita due spazi disposti per lo scontro frontale, due spazi scritti, gestiti e letti da entità in conflitto. Quello che in apparenza potrebbe sembrare una trovata, un divertimento, una divaricazione umoristica della voce narrante, si configura, di fatto, fin dagli esordi, come gioco al massacro. Contini ha

\footnotetext{
27. F. Pedriali, Per Robert Dombroski, in "Edinburgh Journal of Gadda Studies», www.arts.ed.ac.uk/italian/gadda.html

28. C.E. Gadda, Meditazione breve circa il dire e il fare, in I viaggi la morte, cit., p. 444-454.

29. C.E. Gadda, Le belle lettere e i contributi espressivi delle tecniche, in I viaggi la morte, cit., p. 481.
} 
parlato di autonomia del commento; io ho poi irrobustito in negativo il termine per parlare di estraneità. Oggi, parlerei di una struttura di esteriorizzazione o di ricreazione della solitudine della monade, stretta nei suoi tanti ruoli e travestimenti. Questo risulta evidente se si osserva $\mathrm{Il}$ castello di Udine, vera e propria messinscena guerresca, dove si contrastano un'entità che struttura un discorso e una che lo destruttura, non lesinando riprensioni e correzioni, giudizi severi o stroncatori, indebolendo, disgregando, distruggendo quello che l'altra edifica.

Se pensiamo al rapporto fra il testo e il lettore, risulta che questi deve lottare su ben due fronti: con le glosse e con il testo, che queste sembrano spiegare mentre ne pregiudicano la fruibilità. Infatti, volendo considerare la nota dotata di funzione interpretativo-pedagogica come regola nell'ambito di un apparato annotativo, come ci suggerisce Gérard Genette $^{30}$, si osserva che la maggior parte delle note a pie' di pagina integrate nel Castello costituiscono un'ostentata deroga all'uso. Gadda rompe l'intesa fra chi legge e chi scrive, sottolinea l'impossibilità del collegamento armonico, allestendo lo scenario di un vero e proprio corpo a corpo con la scrittura. La scrittura stessa nasce come corpo a corpo e di quel corpo a corpo si nutre per rilanciare le sorti del combattimento. Che così concepisca il proprio lavoro, Gadda lo dice in Come lavoro, appunto: "L'attore del giudizio e la cosa giudicata, lo scrittore e la scritta, il narratore e la narrata, e' stanno fra loro come combattenti in duello, di cui l'uno si creda aver sospinto al muro (acculé au mur) il su' rivale. Il giudizio, la rappresentazione, la Vorstellung (il duello) non può celebrarsi, è ovvio, senza il coesistere e il convenire dei due ${ }^{31}$ ».

Se facciamo un passo indietro, notiamo che sulla logica del duello, del corpo a corpo, che è poi la logica della guerra di trincea, è basata la Meditazione milanese, in cui Gadda non disegna propriamente il campo di battaglia, come farà nel Castello, ma inventa uno «sfidante", il "critico », il cui punto di vista è in perenne conflitto con «il gramo filosofo» che enuncia il proprio pensiero. La schermaglia, che nella prima stesura viene introdotta a partire dal paragrafo $\mathrm{V}$, nella seconda viene anticipata al paragrafo III : in apparenza, Gadda ricorre all'antico schema della disputa filosofica per accedere a rapide concatenazioni di pensieri, per costruire nessi e dissolvere ostacoli, ma noi ci accorgiamo che se non dà un inquadramento conflittuale al discorso, senza scontro, senza diverbio, senza antagonista, senza fuoco nemico, non riesce letteralmente a procedere. La lacerazione,

30. G. Genette, Seuils, Paris, Seuil, 1987.

31. C.E. Gadda, Come lavoro, cit., p. 430. 
la lotta, la rottura non sono sempre così evidenti : gli esempi citati sono tra i più macroscopici, e quindi immediatamente fruibili, perché toccano platealmente la struttura, il punto di vista, la voce ${ }^{32}$.

Non è possibile qui dare conto di altre battaglie testuali di Gadda, per esempio quelle che riguardano la sistemazione dei pezzi da combattimento: Gadda è sempre preso da una battaglia micro- e macrotestuale, deve decidere quali brani eliminare, quali lasciar vivere, quali accantonare provvisoriamente, prigionieri, per poi magari liberarli, e muove continuamente le pedine sulla scacchiera-campo di battaglia della sua opera, come Cesare spostava manipoli, centurie, coorti, legioni. Anche questo passare la vita a dannarsi l'anima su questioni di strategia, in scontri con se stesso, in senso orizzontale-variantistico, in senso verticale-espansivo, che poi faranno la felicità degli esegeti, va capito nell'ottica di colui che è andato verso la scrittura con la sua «identità di ferito, di smarrito, di povero, di "dissociato noetico" ${ }^{33}$ ". E che, per spiegarci come lavora, ci dice: « Rabbia di mozzicato da un cane, lacrime di sangue e di cenere [la cenere delle battaglie, certamente, quella del racconto andato negli Accoppiamenti giudiziosi, n.d.r.] non deterse negli anni. Sputi in faccia, al '19, fino a sentirmi dire, nel '40: "S'io fossi un uomo, sarei già partito volontario ». No, non sono partito volontario a quarantasette anni per la bella guerra del « Se avanzo seguitemi». Sono partito volontario e rivolontario a ventidue, nel giugno del '15; e poi nel maggio del '16, nel luglio '1 $17^{34} »$.

E neppure c'è spazio per parlare del rapporto conflittuale che Gadda instaura con la letteratura tutta intera, ché questo ci porterebbe troppo lontano.

Ma vale la pena di proporre almeno un esempio di insorgenza insospettabile di scrittura-guerra: insospettabile perché di non scontata identificazione. Estraiamo dalla Cognizione del dolore la scenata di Gonzalo a proposito del nipotino del colonnello Di Pascuale:

32. Ovviamente, sappiamo che il duello che Gadda continuamente inscena avviene anzitutto tra sé e sé, in un solo soggetto che sdoppia se stesso e lotta con se stesso, e sappiamo che la biforcazione del discorso per dar spazio alla voce dell'altro in sé può prestarsi a percorsi di lettura di tipo diverso da quello qui proposto, facendo leva su altri protocolli interpretativi. Ma gli uni non escludono gli altri, anzi, dovranno intrecciarsi agli altri, perché la scrittura di Gadda non è una banalità lineare, è un nodo o groviglio, o garbuglio o gnommero, "verso cui - ci dice il narratore del Pasticciaccio - hanno cospirato tutta una molteplicità di causali convergenti». Per questo, pur avendo dato anni or sono (cf. nota 16) un'altra interpretazione del conflitto di voci, ritengo che si deve continuare a leggere e interpretare Gadda anche "per modificare le nostre prime interpretazioni già contestuali in altre interpretazioni contestuali, in cui il testo tuttavia sempre si pone come loro correlato oggettuale contenente un principio regolativo" (E. Garroni, Interpretare, in Il testo letterario. Istruzioni per l'uso, a cura di M. Lavagetto, Roma-Bari, Laterza, 1996, p. 281).

33. C.E. Gadda, Come lavoro, cit., p. 431.

34. Id., p. 429. 
... Ma non è il nipotino del Di Pascuale?, dimandò questi.

... Non so chi sia, né di chi sia il nipote... Quel che so è che mia madre è rimbambita... come tutti i vecchî.... : parlò concitatamente. Il dottore si batteva il polpaccio con la bacchetta. ... che ha bisogno di bavare bontà sul primo vitello che le càpita tra i piedi... sul primo cane randagio che viene a oltre... Anche i nipoti dei colonnelli in vacanza, adesso... da fargli ripetere choux, bijoux, cailloux, poveri tesori... Perché si diventa buoni, buoni!. Gridava. Pareva ammattire. «Buoni, buoni!... si diventa... Fino a che i gerani, le màmmole, ci premiano della nostra buona condotta... Della bontà definitiva... ${ }^{35}$

Manzotti commenta: «choux... cailloux: Sono le tre prime eccezioni (seguono genou, joujou, hibou et pou) alla formazione regolare (in $-s$ ) del plurale nelle parole che terminano per - ou; cfr. p. 198 rr. 174-176 [...] e p. 289 rr. 370-71 [...]» (p. 164n).

Come suggerisce Manzotti, andiamo a p. 198, dove la tiritera di «choux... cailloux» affiora in questi termini :

... Ma li regala al nipotino color caffe... Perché si degni di leggicchiare quel po' di gufi e di càvoli con la x... D'imparucchiare quattro parole sbagliate... Con quella bella pronunzia che ha, il nipotino... Mi vien male solo a sentirlo:

Maître corbeau sur un arbre perché... Oh! nolite margaritas. Del La Fontaine a uno scemo simile. E mia madre, mia madre! E gli regala i fichi, le pesche, le caramelle...

Sempre seguendo Manzotti, andiamo a p. 289, dove essa riappare puntualmente:

I ragazzi, poi, sembrava addirittura che li avesse in odio. Una severità cupa gli si metteva sulla faccia a trovarne in casa anche uno solo, come quel povero scioccherello, sorrise la madre, del caillou, bijou.

Notiamo en passant che la tiritera viene proposta da vari punti di vista, per giunta tra loro contrastanti. Nei primi due casi da quello di Gonzalo, con tonalità diverse: entrambe le volte Gonzalo parla con il medico, dapprima concitato e in preda a "un'ira incredibile» che altera «la sua fisionomia incoerente» (p. 165), tanto che il narratore dice: "Gridava. Pareva ammattito"; e poi, essendo alle variazioni definite "estrose» subentrato il dolore che aveva dissolto "gli anni irripetibili» a fronte dei quali «rimaneva la morte», ecco che Gonzalo rilancia l'invettiva. Nel terzo e ultimo caso abbiamo la percezione della señora vista dal narratore che nega quella del figlio evidenziandone la difformità, l'enormità: poiché, contrariamente a quel che lui crede, per lei il nipotino del colonnello non è altro che "quel povero scioccherello del caillou, bijou» al quale pensa con un sorriso non più che condiscendente. Così, l'episodio dei plurali irregolari in $-\mathrm{x}$, costruito per trascinamenti, restituisce l'essenza dei tre conflitti

35. C.E. Gadda, La cognizione del dolore, edizione critica a cura di E. Manzotti, Torino, Einaudi, 1987, p. 164. D'ora in poi, le indicazioni delle pagine citate verranno date tra parentesi nel testo. 
portanti del romanzo: Gonzalo-madre, Gonzalo-mondo, GonzaloGonzalo.

Ma non basta: c'è una coda. Perché mai questa vétille sta al centro dei pensieri di Gonzalo, a tal punto che anche la madre la rammenta, a tal punto che chi scrive la reitera per ben due parti e tre tratti del romanzo, facendola così consistere come oggetto mentale significativo nel tempo del racconto e nel tempo raccontato? Osserviamo anzitutto che tutte e tre le volte la tiritera insorge in un contesto in cui viene richiamata la guerra: la prima volta, mentre la madre è al cimitero per deporre fiori sulla tomba dell'altro figlio, quello morto in guerra, e arriva il ragazzino che Gonzalo caccia via a male parole. Quando il dottore gli chiede: "Ma non è il nipotino del colonnello Di Pascuale?», Gonzalo risponde dapprima: "Non so chi sia, né di chi sia il nipote..." e, tre battute dopo: "anche i nipoti dei colonnelli in vacanza, adesso...» (per Gonzalo i colonnelli possono far tutto tranne che essere in vacanza, ovviamente). La terza volta, quando la madre rammenta la fissazione di Gonzalo sulla tiritera, questo suo ricordo ci viene presentato sulla scia di quello che precede, che è riferito al ritorno di Gonzalo dalla guerra:

Era incolume, con poveri anni dentro le grigie controspalline del ritorno. Forse la sua guerra, a
lui, non era stata pericolosa. Non raccontava nulla, mai : non ne parlava ad alcuno: non certo
ai ragazzi, se lo attorniavano in un minuto di lor sosta, belligeranti o ammiragli sgraffiati, accal-
dati, con baionette di latta: e nemmeno alle signore in villa, ch'erano, diceva, tra le più elette
gentildonne di Pastrufazio le più assetate di epos: e in conseguenza le più entusiaste bevitrici di
fandonie (p. 288-289).

Associazione mentale curiosa, ma non del tutto, come sa chi si è già soffermato a riflettere sulla seconda insorgenza della tiritera, la più importante, perché ci porge la chiave del mistero. In questa circostanza, Manzotti ha giustamente chiosato: "gufi... con la $x$ : E' già in CU, 70 (Castello di Udine, n.d.r.) "alcuni parrucchieri molli ripetevano choux, bijoux, cailloux", come stereotipo di faticato e ottuso apprendimento della lingua» (p. 198n). Ma leggendo questo brano della Cognizione ricordiamo che abbiamo trovato un'aggiunta: "Con quella bella pronunzia che ha, il nipotino... Mi vien male solo a sentirlo: Maître corbeau sur un arbre perché... Oh! Nolite margaritas. Del La Fontaine a uno scemo simile. E mia madre, mia madre!». E, se andiamo a leggere avanti nel Castello, se cioè non ci accontentiamo del lacerto evocato da Manzotti nella sua nota, troviamo quanto segue: "e pochi minuti dopo La Fontaine, inimitabile e chiara eleganza parlava già come il groom dell'Albergo del Gallo, all'arrivo degli sposi di Brembate supposti svizzeri.»Questi e non altri gli agganci centrali: La Fontaine e la pronuncia storpiata, cioè le colpe dell'insegnante. Chi è l'insegnante? "Mia madre, 
mia madre!», naturalmente, nella Cognizione del dolore. E nel Castello di Udine?

Un insegnante di francese, territoriale dai baffi, temperamento di ginevrino. Dettava a Rastatt [il primo campo di prigionia] le sue sentenze formative de' più rari plurali, con la sicurezza secca di chi governa, al circo, un numero di cani ammaestrati ${ }^{36}$.

Ed ecco le parole più importanti: di costui Gadda dice che fu «il feticcio della mia ira". Il feticcio della mia ira - questo l'oggetto e insieme la figura essenziale che vengono trasferiti dal Castello alla Cognizione, senza soluzione di continuità, dalla vicenda testimoniata alla finzione che ormai la rende pressoché inidentificabile, visibile solo in filigrana, perché nel frattempo una rielaborazione è avvenuta (teniamo presente che i due testi sono pressoché contigui: Imagine di Calvi infatti è del '32). Non dirò che a Lukones esplode l'ira covata fin da Rastatt, perché non mi occupo della vita di Gadda. Dirò invece che Gadda rielabora un ricordo di guerra quale esso è emerso in prima istanza in pagine di guerra. E lo fa:

1) per darsi il «la»: visto che ha bisogno di un'ira che sia un'ira, recupera intero uno scenario di ira potente già testualmente produttivo;

2) per ascriverla a Gonzalo, a colui cioè di cui costruisce la personalità usando questa e tante altre occasioni tratte dai testi di guerra.

Gli imperdonabili errori di un territoriale baffuto diventano quelli della señora, i parrucchieri il ragazzino che sta lì a imparucchiare; l'occasione, in compenso, quella dei plurali irregolari in -x, della storpiatura di La Fontaine, rimane inalterata, perfino nella sottolineatura degli orroridelizie della pronuncia errata: rammento che nel Castello per i parrucchieri, come nella Cognizione per il nipotino del colonnello, viene segnalato un punto ostico particolare: laddove i parrucchieri inciampano su «bocù » dicendo "bocü », così in bocca allo sventurato ragazzino "scie» diventa «chie».

"Pace non conosceva Gonzalo» (p. 313). E questo, va da sé, perché ha l'esaurimento nervoso, è preso "nella turpitudine pazza» (p. 301) della sua ira, è un anomalo psichico (come il protagonista della Cenere delle battaglie), è un dissociato noetico (come Gadda dice di sé). Ma anche perchè vale la petizione che ammette ciò che l'enunciato nega: guerra conosceva Gonzalo. La conoscenza di Gonzalo, la sua cognizione, passa tutta attraverso la guerra. L'ira e la guerra, l'ira della guerra, l'ira-guerra: questa la stoffa di cui è fatto Gonzalo. 
Per concludere, vale la pena di ribadire che il Giornale di guerra e di prigionia e Il castello di Udine sono testi-serbatoio ai quali tornare a rifornirsi di continuo. Come abbiamo visto, siamo ben oltre la pura e semplice scorta di temi e motivi: abbondano rivoli e flussi sotterranei, non ancora tutti noti. Uno di questi, già identificato ma di cui si parla poco, forse perché sembra aberrante farlo, andrebbe fatto affiorare. Mi riferisco al filone delizie della guerra e all'appagamento del desiderio che la scrittura-guerra porta con sé (potremmo dire tornaconto della scrittura-guerra nel senso in cui Freud parlava di tornaconto della malattia), che bisognerebbe integrare in una struttura globale di rilettura dell'ampio progetto conoscitivo rappresentato dall'opera gaddiana.

Rammento per adesso un paio di occasioni testuali significative, su cui sarà utile tornare a meditare in altra sede:

Di certe ore di guerra [...] non dirò lo ringrazio (il Padre Eterno), è bestemmia, dirò solo che le ho vissute con orgoglio e con gioia;

In guerra ho passato alcune ore delle migliori di mia vita, di quelle che m'hanno dato oblio e compiuta immedesimazione del mio essere con la mia idea: questo, anche se trema la terra, si chiama felicità ${ }^{37}$

37. Su questo argomento, almeno due riferimenti critici: C. Mileschi, "La guerra è cozzo di energie spirituali»: estetica ed estetizzazione della guerra in Carlo Emilio Gadda, in "Bollettino 900", 2003, http://www2.unibo.it/boll900/numeri/2003-i/Mileschi.html e F.G. Pedriali, The Mark of Cain: Mourning and Dissimulation in the Works of C.E. Gadda, in Carlo Emilio Gadda: Contemporary Perspectives, edited by M. Bertone \& R.S. Dombroski, Toronto-London, The University of Toronto Press, 1997, p. 132-158. 